\title{
On Two Cases of Abdominal Pregnancy.
}

By John Benjamin Helluer, M.D. (Lond.), Hon. Obstetric Physician to the General Infirmary at Leeds, and Lecturer on Diseases of Women and Children in the Yorkshire College.

I.

A CASE of abdominal pregnancy secondary to partial rupture of tubal gestation sac; abdominal section 15 months after conception and 8 months after death of fœtus.*

Summary. This patient had seven months amenorrhœa with signs of pregnancy, and was confined to bed with pain and symptoms attributed to "inflammation." The pain subsided and the health improved, and menstruation recommenced and became excessive; eight months later a dead fœtus was removed by abdominal section from the peritoneal cavity, together with a tumour which arose from the right oviduct and contained the placenta. The fotus was closely covered by an amniotic sac, which was connected with the tubal tumour. Recovery without complication. Photograph of specimen reproduced in Fig. I.

Clinical history. B.0., married, aged 30, was admitted into the Leeds Infirmary on September 10th, 1903, for abdominal tumour; she stated that her father died of phthisis and her mother of cancer of the breast. She herself enjoyed good health till the present illness. She had a still-born child eight years ago, a living child six years ago, and another a year later; after this she had no pregnancy till 13 months before admission. Then she ceased to menstruate and had signs of pregnancy; she suffered much from vomiting and severe abdominal pain. She says that she was confined to bed for three months, and was supposed to have inflammation, the body being swollen and the pain severe. Subsequently the pain subsided, the abdomen became less swollen and menstruation recommenced after seven months amenorrhoea. She was now able to get up and felt well, but sought advice for menorrhagia. On admission she was found to be in good condition with florid complexion and healthy aspect. Heart, lungs and kidneys normal.

Abdominal examination. The walls were fairly thick, the abdomen was somewhat prominent, especially on the left side; on palpation a somewhat hard and irregular swelling was felt extending

\footnotetext{
* Case communicated to the Obstetrical Society of London, October 7th, 1903.
} 
from the pelvis to the level of a point half way between the umbilicus and the sternum. At its upper part it lay principally to the left of the median line, but it occupied a more central position below. There was a small hard nodule at the navel. The upper part of the tumour seemed to be in some degree differentiated from the pelvic part and was harder. The tumour was somewhat mobile, but the lower part was more fixed. There was dulness over the tumour, but the flanks were resonant. No tenderness on pressure or pain on manipulation.

On vaginal examination the cervix appeared to be of normal consistence and not unduly patulous. In the anterior fornix a rounded tumour could be felt lying in front of the uterus. It was continuous with the pelvic portion of the abdominal tumour. To the touch it was soft and somewhat elastic.

Operation, September 12th, 1903. The patient was anæsthetised with ether. On exploring the abdomen by a median incision a dead extra-uterine foetus was exposed directly the peritoneum was opened. It lay with the head above and the back behind; the knees flexed on the thighs and the thighs on the abdomen. The nodule which had been felt at the navel was the right knee. The fotus was compressed by a tightly-fitting capsule of membrane, which was semi-transparent and which seemed to be the amniotic sac. There was no liquor amnii. The arms were bound down to the sides and the features were flattened out as appears in Fig. I. The sac had adhesions externally to the mesentery and omentum. These adhesions were separated without difficulty, some being ligatured and a portion of the omentum removed. On drawing the fœtus out by the upper end the legs became extended, the amniotic sac giving way around the waist. It was then found that the right foot was attached to a pelvic tumour which lay in the utero-vesical pouch and on the right side. This was enucleated rather easily, some adhesions being broken down and some tied and divided; these last included some processes of the broad ligament, but the tumour was not in the broad ligament. There was not much bleeding, but there was a little general oozing from the site of some of the pelvic adhesions. A glass drainage tube was left in the pouch of Douglas. The abdominal wound was closed with continuous catgut suture of peritoneal edges, and interrupted silk-worm gut sutures through skin and muscle, a few interrupted catgut sutures being used to make the edges of the aponeurosis meet accurately. There was not the least suggestion of any septic process in the abdomen or in the parts removed. The fœtal tissues seemed rather brittle. The fœetus was removed with the pelvic tumour hanging from its foot, and this tumour contained the placenta. 
After history. The patient made a rapid recovery without complications. No vomiting after operation. No morphia needed. Tube removed on the fourth day; union by first intention. The temperature was practically normal from the third day onwards. Sent to convalescent home on October 3rd.

Description of parts removed. The fœtus is a male with wellformed genitals; weight, 2lbs. 7ozs.; length, $12 \frac{1}{2}$ ins. It corresponds to between 25 and 28 weeks development. It is free from deformity and must have been dead for some months. It is enveloped as far as the waist in a membranous sac which fits closely over head and trunk, binding the arms closely to the sides, and in the face flattening the features; this sac appears to be the amnion. There is no liquor amnii but the sac can be reflected without difficulty from the skin of the fotus. The legs are now extended, and the front of the abdomen and thighs are not covered by amnion, and the skin here is redder and softer than in the parts immediately beneath the amnion. On flexing the legs and thighs the continuity of the amniotic sac is restored and it is seen to be continued over the buttocks and the rest of the foetus. A stump of umbilical cord hangs from the umbilicus; the cord was torn when the legs were extended during the extraction of the foetus. The pelvic tumour has the appearance of a distended oviduct and is about the size of a closed fist. The fimbriated extremity of the oviduct is clearly seen, and the outer two or three inches of the oviduct can be traced in the wall of the tumour at the upper and inner part. The right foot of the fotus is embedded in the upper side of the tumour, but by a little dissection it is easy to see that the foot has an amniotic investment and that it is really outside the main capsule of the tumour, although embedded in an invagination thereof. With a little force it could be drawn out and separated. The continuation of the umbilical cord enters the tumour near this foot. A probe passes two or three inches along the oviduct and then enters the substance of the tubal sac. On section there is a wellmarked capsule with which the amnion is blended above. The section of the tumour is uniform, reddish in colour, fibrous, and somewhat granular looking. The contents of the sac are the placenta in an altered and degenerated condition.

On microscopic examination the substance of the tumour shows fibrous tissue with remnants of chorionic villi and some calcareous particles. The sac wall, according to the report of the pathological committee of the Obstetrical Society is made up partly of the ampullar end of the tube, but mainly of new fibrous tissue. The case is an example of tubo-abdominal pregnancy. 


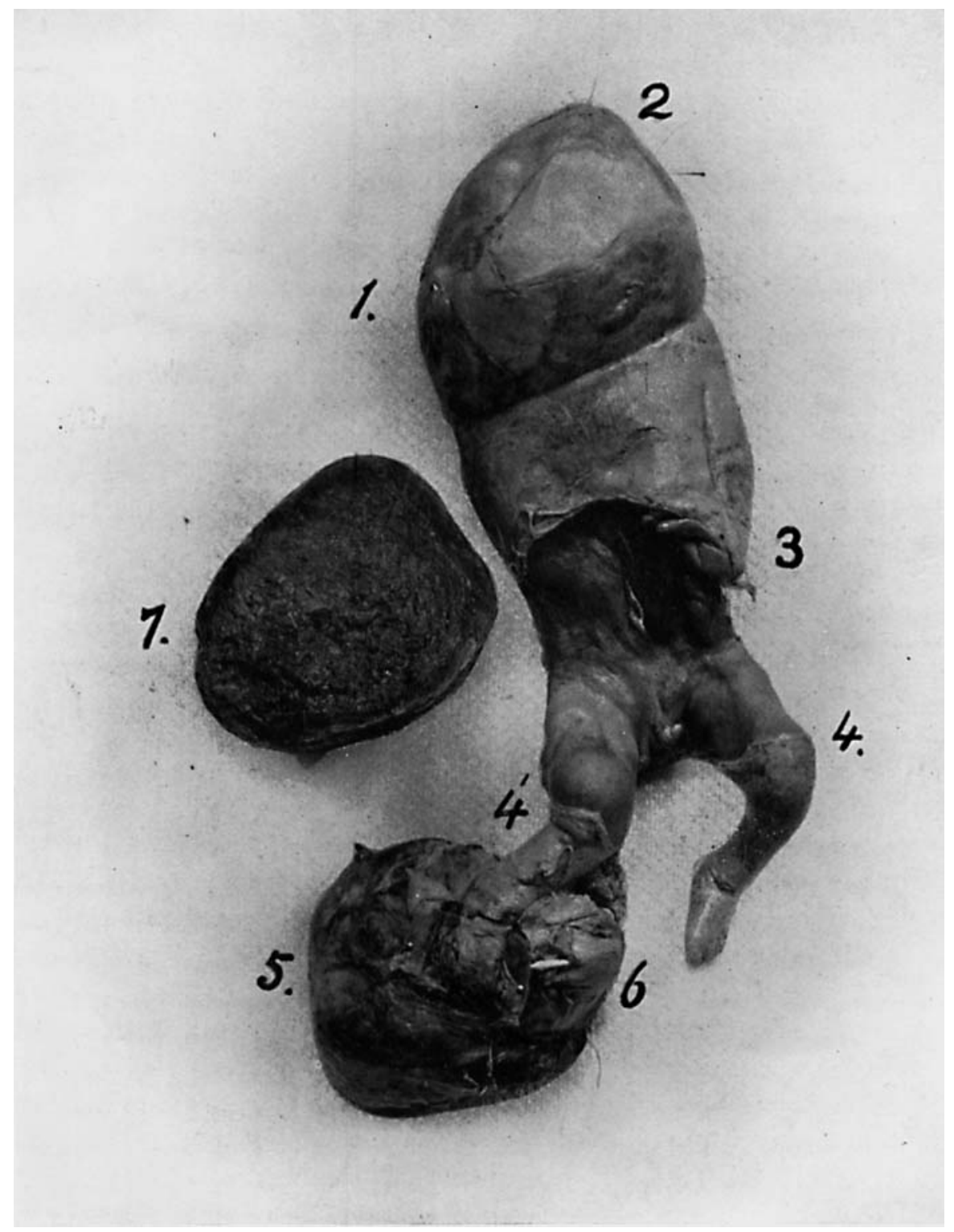

Frg. I 


\section{Description of Fig. I.}

1, 2, 3. Upper half of foetus covered by amniotic sac.

3. Shows left hand and torn edge of amnion.

4, 4.1 Show continuation of amnion.

5, 6. Tubal sac.

6. Shows dorsal end of oviduct, a white probe is placed in ostium abdominale. Two small flaps of capsule are pinned open to show foot.

7. Posterior half of tumour to show section. Note capsule.

This case may be compared with a very similar one communicated by Mr. Lawson Tait to the London Obstetrical Society in 1892, and which is quoted by Mr. T. W. Taylor in his valuable work on " Extrauterine Pregnancy" (London: 1899, p. 57). The present case is a further illustration of the fact that intra-ligamentous rupture is not a necessary condition of secondary abdominal pregnancy, as Mr. Taylor well points out.

II.

A specimen of uterus with rudimentary horn from a case where abdominal pregnancy occurred 19 years before.

The patient from whom this specimen was obtained has recently died in the Leeds Infirmary from strangulated ventral hernia. I am indebted to my colleagues, Mr. Littlewood, who had the case under his care, and Dr. Wardrop Griffith, who made the post-mortem examination, for the opportunity of examining the specimen and recording the case. Dr. Braithwaite has also given me information concerning the operation. At a meeting of the Obstetrical Society of London, January 13, 1886, Dr. James Braithwaite published " A case of gastrotomy for extra-uterine gestation in which the placenta never came away" (Trans. Obstet. Society, vol. xxviii., p. 33.) The operation was performed in December, 1884. The fœtus, which was full-grown, had been dead about 3 weeks. We quote Dr. Braithwaite's description of the operation. "It turned out that the fœtus was quite free in the abdominal cavity, except that a fine transparent membrane appeared to shut off the intestines above from the fotus below. There was, however, nothing of the kind visible in the lower part of the abdominal cavity. The large placenta fitted the top of the uterus like a cap, and was spread out on its posterior surface and the neighbouring parts. The wound was closed except at its lower 
angle, where the funis was left hanging out, and also a drainage tube ... The recovery was perfect, but rather slow. The curious and, $I$ believe, unique point in the case was that the placenta never came away, except a morsel about 20 grains in weight, which protruded at the upper angle of the wound on the 6th day." Mr. Knowsley Thornton, commenting on the paper at the time, said that if the placenta had really been absorbed by the peritoneum, it was one of the most remarkable pathological facts ever recorded. Some discussion on the probable fate of the placenta followed. Fourteen months later Dr. Braithwaite made a bimanual examination, and could find no indication of the existence of the placenta except a small ring of dense tissue behind the uterine fundus. The patient at the time of operation was about 43 . She had borne living children previously, but had no further pregnancy after this operation.

The patient was admitted into the Leeds Infirmary on February $6 \mathrm{th}, 1904$, at the age of 62 , suffering from strangulation in a large ventral hernia. She was in a bad condition, and in spite of immediate operation she died on February 8th. At the post-mortem examination various adhesions were found round and about the old abdominal wound and the hernial region. The pelvis, however, seemed normal; no trace of the old gestation or of the placenta was found, and it was only after the uterus and its appendages had been removed from the body that the uterus was seen to be abnormal. The uterus and appendages were then handed over to me.

The following description of the parts will be better understood if reference be made to the excellent drawing of the specimen, which is here reproduced.

Description of uterus and appendages. The parts submitted consist of the uterus and appendages, together with the upper part of the vagina $\left(1,1^{1}\right)$. The uterus has been laid open by a longitudinal incision extending from the os internum to the right cornu (5). The uterus measures externally in the middle line about $3 \frac{1}{2}$ ins. It is bicornute and unsymmetrical. The right cornu (5) is well developed and the uterine cavity is continued into it to the ostium internum of the oviduct. The left cornu $\left(5^{1}\right)$ is rudimentary in its development. It is smaller than the right, it is club-shaped, it is impervious in the greater part of its length, but a transverse incision made near the outer end $(6,6)$ shows a small limited cavity containing caseous material $\left(6,6^{1}\right)$. This cavity does not extend along the cornu, and there is no sign in the uterus of any communication with the cornu. 


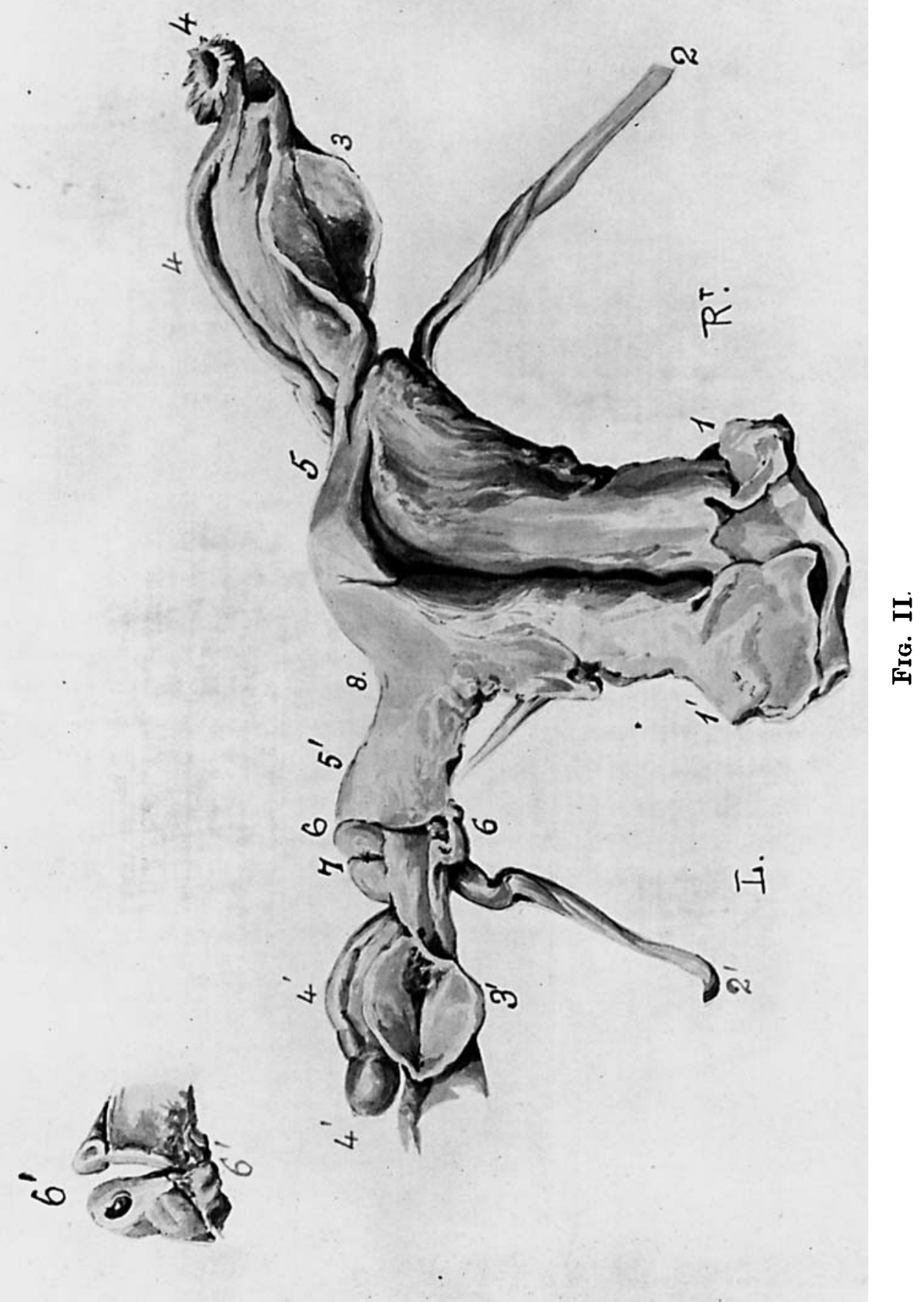


If any central canal ever existed it has become obliterated. A wide rotch (8) marks the divergence of the two cornua.*

At the outer end of the rudimentary horn is a well-marked cicatrix (7). To this we draw special attention as the probable seat of an old rupture. The two round ligaments are well marked on each side $\left(2,2^{1}\right)$ and show the termination of each cornu. The right oviduct $(4,4)$ is quite normal and terminates in a normal fimbriated end. The right ovary (3) has been cut open longitudinally. It shows almost no trace of Graäfian follicles and is in the atrophic condition common at her age (62). The meso-salpinx is thin and transparent, and normal. The left ovary ( $\left(^{1}\right)$ is smaller than the right and measures about 1 in. by $\frac{1}{2} \mathrm{in}$. The left oviduct $\left(4,{ }^{1} 4^{1}\right)$, shorter than the right, measures 3ins. long, and its fimbriæ are adherent, so that the end is clubbed, but there is very little thickening. The left meso-salpinx is thin and transparent. The broad ligaments on each side were normal.

Remarks. The most probable explanation of this case is that it was an example of pregnancy in a rudimentary cornu, that rupture took place gradually, the amniotic sac remaining entire, and the placenta gradually extending over the uterine fundus and the adjacent parts.

The thin membrane which Dr. Braithwaite describes was almost certainly amnion. The complete absorption of the placenta is a very important point. The fact that both oviducts were normal as well as the meso-salpinx on each side makes it very unlikely that tubal pregnancy had been present. The fact that a rudimentary cornu showing an old scar was found makes it probable that the pregnancy was cornual, becoming abdominal. We might describe the case as one of cornu-abdominal pregnancy. We do not know whether the term cornu-abdominal has been previously employed, but it seems legitimate in this case.

\section{Description of Fig. II.}

Uterus and appendages removed post mortem, posterior aspect. An incision has been made along posterior uterine wall and another across the left cornu $(6,6)$.

- Specimens very similar to this have been described as unicornute uteri. As regards the cavity of the fundus the term is applicable. But the uterus has two horns, and is therefore bicornute; one of the horns is rudimentary, but yet it was sufficiently developed to become the seat of a pregnancy. We cannot bring ourselves to describe the specimen as a one-horned uterus in which pregnancy occurred in the other horn! 
444. Journal of Obstetrics and Gynocology

1, 1.1 Vagina at level of os externum uteri.

2, 2.1 Round ligament.

3, 3.1 Ovaries cut longitudinally.

4, 4. Right oviduct ending in normal fimbriated extremity.

$4,{ }^{1} 4 .^{1}$ Left oviduct ending in clubbed extremity.

5. Right uterine horn.

6.1 Left uterine horn.

6,6. Section through left horn (closed).

6, ${ }^{1}$ 6.1 Same opened out showing small cavity.

7. Cicatrix, showing seat of old rupture.

8. Bifurcation of uterine cornua.

For the photograph we are indebted to Mr. Oliver, of the Leeds Infirmary, and for the drawing of case No. 2 to Miss Ethel Wright. 